\title{
DNA methyltransferase DNMT1 inhibits lipopolysaccharide-induced inflammatory response in human dental pulp cells involving the methylation changes of IL-6 and TRAF6
}

\author{
LUHUI CAI* , MINKANG ZHAN*, QIMENG LI, DI LI and QIONG XU \\ Guanghua School of Stomatology and Guangdong Provincial Key Laboratory of Stomatology, \\ Sun Yat-sen University, Guangzhou, Guangdong 510055, P.R. China
}

Received May 7, 2019; Accepted November 5, 2019

DOI: $10.3892 / \mathrm{mmr} .2019 .10860$

\begin{abstract}
Dental pulp inflammation is a pathological process characterized by local lesions in dental pulp and the accumulation of inflammatory mediators. DNA methylation of cytosine residues is a key epigenetic modification that is essential for gene transcription, and plays pivotal roles in inflammatory reactions and immune responses. However, the function of cytosine DNA methylation in the innate immune defense against the inflammation of dental pulp is poorly understood. To investigate the effect of DNA methylation in inflamed dental pulp upon innate immune responses, expression levels of the DNA methyltransferases (DNMT1, DNMT3a and DNMT3b) in human dental pulp cells (hDPCs) after lipopolysaccharide (LPS) stimulation were evaluated by western blotting and reverse transcription-quantitative (RT-q) PCR. Only DNMT1 expression was decreased, while the transcription of inflammatory cytokines was increased. In the immune responses of LPS-induced hDPCs, the results of RT-qPCR and ELISA showed that DNMT1 knockdown promoted the production of the pro-inflammatory cytokines, interleukin (IL)-6 and IL-8. Western blotting demonstrated that DNMT1 knockdown increased the phosphorylation levels of IKK $\alpha / \beta$ and $\mathrm{p} 38$ in the $\mathrm{NF}-\kappa \mathrm{B}$ and MAPK signaling pathways, respectively. Furthermore, MeDIP and RT-qPCR analysis demonstrated that the 5-methylcytosine levels of the $I L-6$ and TNF receptor-associated factor 6 (TRAF6) promoters were significantly decreased in DNMT1-deficient hDPCs.
\end{abstract}

Correspondence to: Professor Qiong Xu, Guanghua School of Stomatology and Guangdong Provincial Key Laboratory of Stomatology, Sun Yat-sen University, 56 Ling Yuan Xi Road, Guangzhou, Guangdong 510055, P.R. China

E-mail: xqiong@mail.sysu.edu.cn

*Contributed equally

Key words: DNA methyltransferase, methylation, inflammation, lipopolysaccharide, dental pulp
Taken together, these results indicated that the expression of DNMT1 was decreased after LPS stimulation in hDPCs. DNMT1 depletion increased LPS-induced cytokine secretion, and activated $\mathrm{NF}-\kappa \mathrm{B}$ and MAPK signaling; these mechanisms may involve the decreased methylation levels of the $I L-6$ and TRAF6 gene promoters. This study emphasized the role of DNMT1-dependent DNA methylation on the inflammation of LPS-infected dental pulp and provides a new rationale for the investigation of the molecular mechanisms of inflamed dental pulps.

\section{Introduction}

Dental pulp inflammation is a pathological process characterized by various bacterial virulence factors that often elicits a dental emergency, and may develop into periapical disease or pulp necrosis (1). Lipopolysaccharide (LPS) is commonly released from gram-negative bacteria. When LPS enters the dental pulp, it can evoke an inflammatory response; LPS is also closely associated with pulpitis and periapical periodontitis (2). Previous studies have found that LPS can stimulate Toll-like receptor (TLR) 4 in the cell membranes of human dental pulp cells (hDPCs), and activate the NF- $\mathrm{B}$, ERK1/2 and p38 pathways, thereby producing inflammation-related cytokines, including interleukin (IL)-6 and IL-8 $(3,4)$. Although there are a number of mechanisms associated with the development of dental pulp infection, the specific molecular mechanism is still unclear $(5,6)$. Recent studies have suggested that epigenetic alterations are crucial regulators in the occurrence and development of dental pulp infection (7-9).

DNA methylation that occurs at cytosine-phosphate-guanine (CpG) dinucleotide sites is the most common epigenetic modification event in the genome (10). The DNA methylation process involves placing a methyl group onto the 5-position of cytosines situated in $\mathrm{CpG}$ dinucleotides and turning the cytosine into 5 -methylcytosine $(5 \mathrm{mC})$, which is catalyzed by members of the DNA methyltransferase (DNMT) family (11). DNMT1 can methylate hemimethylated CpGs and is a well-known maintenance methyltransferase that can preserve methylation patterns during DNA replication (12). DNMT3a and DNMT3b are de novo methyltransferases that can methylate unmethylated 
and hemimethylated DNA, and establish DNA methylation patterns in embryo development (13). The roles and functions of DNA methylation patterns have attracted extensive attention, but there has been particular emphasis on their roles in the pathological processes of cancer (14). Only recently have studies begun to shed light on the contribution of DNMTs to the initiation and progression of inflammatory diseases (15). A study on inflamed peripheral blood mononuclear cells (PBMCs) showed that DNMT1 expression decreased after treatment with LPS; DNMT1 modulated the methylation level of gene promoters, thus mediating the transcription of pro-inflammatory cytokines, including IL-6, IL-8 and tumor necrosis factor- $\alpha$ (TNF- $\alpha$ ) (16). In macrophages, DNMT1 contributes to the hypermethylation of suppressor of cytokine signaling 1, a negative regulator of cytokine signal transduction, thereby enhancing the secretion of pro-inflammatory cytokines induced by LPS indirectly (17). DNA methylation could also affect inflammatory reactions by modulating the activation levels of crucial proteins of the $N F-\kappa B$ and/or MAPK pathways $(18,19)$. In addition, DNA methylation epigenetically regulates the transcription of TLRs and signal transduction molecules, including TNF receptor-associated factor 6 (TRAF6) and myeloid differentiation primary response 88 (MyD88). This suggests that DNA methylation is engaged in signaling pathways related to inflammation $(20,21)$. These studies provide evidence indicating that DNA methylation can epigenetically regulate inflammatory reactions via several different mechanisms. However, whether DNA methylation is involved in the modification of dental pulp immunity remains unclear.

Preliminary experiments by our lab showed that in LPS-treated hDPCs, 5-aza-2'-deoxycytidine (5-Aza-CdR), a DNA methyltransferase inhibitor, increased the production of several inflammation-related cytokines (unpublished data). The present study aimed to investigate the effect of DNMT1 on the LPS-induced inflammatory response in hDPCs, thereby exploring the role of DNA methylation in dental pulp inflammation. The results demonstrated that DNMT1 knockdown promoted the expression of pro-inflammatory cytokines and the phosphorylation of IKK $\alpha / \beta$ and p38 in LPS-treated hDPCs. Moreover, DNMT1 depletion decreased the $5 \mathrm{mC}$ level in the $I L-6$ and TRAF6 promoters. These data suggested that DNMT1 may be involved in inhibiting the LPS-induced inflammatory response in hDPCs.

\section{Materials and methods}

Isolation and culture of hDPCs. Healthy permanent premolars and third molars were collected from donors aged 18 to 25 for orthodontic reasons from the Department of Oral and Maxillofacial Surgery, Guanghua School of Stomatology, Sun Yat-sen University for approximately one year between March 2018 and April 2019. Only healthy teeth without carious disease or hyperemic pulp tissue were selected. A total of 128 teeth from 58 donors (29 males and 29 females) were obtained for dental pulp tissue isolation and cell culture. hDPCs were isolated and cultivated using an enzymatic method as described by Gronthos et al (22). After extraction, the teeth were washed with $70 \%$ ethanol and PBS (pH 7.4) and then split open to expose the pulp chamber. The dental pulp tissue was gently isolated with forceps and minced into small pieces, which were then digested in $3 \mathrm{mg} / \mathrm{ml}$ collagenase type I (Gibco; Thermo Fisher Scientific, Inc.) for $20 \mathrm{~min}$ at $37^{\circ} \mathrm{C}$. Subsequently, the minced pulp tissue was cultured in DMEM containing 20\% FBS, $100 \mathrm{mg} / \mathrm{ml}$ streptomycin and $100 \mathrm{U} / \mathrm{ml}$ penicillin (all purchased from Gibco; Thermo Fisher Scientific, Inc.) at $37^{\circ} \mathrm{C}$ with $5 \% \mathrm{CO}_{2}$. The medium was changed every 3 days. When the cells reached $80 \%$ confluence, they were detached using trypsin/EDTA (Gibco; Thermo Fisher Scientific, Inc.) and subcultured at a ratio of 1:2. Generally, 2-3 teeth from one donor were used for each primary culture. For each primary culture, $\sim 10^{6}$ cells at the zero passage were obtained. All experiments were performed with cells from passages two or three. To avoid inter-individual variation, the experiments were performed at least three times for each sample and each experiment, and average data were generated. For each parameter, experiments were replicated three times each using donor cells from three samples, and average data for the three different cell types were obtained.

Treatment with LPS. hDPCs were stimulated for the indicated times $(0,3,6,12$ and $24 \mathrm{~h})$ with $1 \mu \mathrm{g} / \mathrm{ml}$ purified Escherichia coli (E. coli) LPS (Sigma-Aldrich; Merck KGaA) at $37^{\circ} \mathrm{C}$ with $5 \% \mathrm{CO}_{2}(4,23)$. The blank controls were cells without LPS stimulation.

DNMT1 small interfering RNA (siRNA) transfection. siRNA was used in hDPCs to knockdown DNMT1. A total of 3 siRNA sequences (Invitrogen; Thermo Fisher Scientific, Inc.) were designed to target the human DNMT1 gene. Before transfection, hDPCs were seeded in 6-well plates in $2 \mathrm{ml}$ of $\alpha$-MEM at $4 \times 10^{5}$ cells/well containing $10 \%$ FBS for $24 \mathrm{~h}$. After attachment overnight, hDPCs were then transfected with siRNA $(50 \mathrm{nM})$ against DNMT1 or a nontargeting siRNA control using Lipofectamine ${ }^{\circledR} 3000$ (Invitrogen; Thermo Fisher Scientific, Inc.) according to the manufacturer's protocols. After incubation for $24 \mathrm{~h}$, the media was changed, and DMEM supplemented with $10 \%$ FBS was added with or without $1 \mu \mathrm{g} / \mathrm{ml}$ E. coli LPS. All siRNA sequences are listed in Table I. siRNA \#1 with the best interference effect was selected as the DNMT1 target sequence for the subsequent experiments.

Reverse transcription quantitative $(R T-q) P C R$. Cells were lysed using TRIzol ${ }^{\circledR}$ reagent (Invitrogen; Thermo Fisher Scientific, Inc.) following the manufacturer's protocols, and RNA was extracted and reverse transcribed into cDNA with a RevertAid First Strand cDNA Synthesis kit (Fermentas; Thermo Fisher Scientific, Inc.). PCR was performed using the complementary DNA as a template. SYBR-Green I (Roche Diagnostics) RT-qPCR results were detected by a LightCycler ${ }^{\circledR} 480$ thermal cycler. Thermal cycling conditions consisted of initial denaturation at $95^{\circ} \mathrm{C}$ for $5 \mathrm{~min}$, followed by 45 cycles of $95^{\circ} \mathrm{C}$ for $10 \mathrm{sec}, 65^{\circ} \mathrm{C}$ for $20 \mathrm{sec}$ and $72^{\circ} \mathrm{C}$ for $30 \mathrm{sec}$. The relative results were normalized to the $G A P D H$ mRNA levels (24). The primer sequences were designed using Primer Express Software v3.0.1 (Thermo Fisher Scientific, Inc.) and are listed in Table II.

Western blot analysis. Protein was extracted from hDPCs using RIPA buffer (Beyotime Institute of Biotechnology), and the concentrations were detected using a BCA Protein Assay kit 
Table I. Sequences used for DNMT1 siRNA.

\begin{tabular}{ll}
\hline DNMT1 siRNA & \multicolumn{1}{c}{ Sequence $\left(5^{\prime}-3^{\prime}\right)$} \\
\hline \#1 siRNA & Sense: GGGACUGUGUCUCUGUUAUTT dTdT \\
\#2 siRNA & Antisense: dTdTAUAACAGAGACACAGUCCCTT \\
& Sense: GCACCUCAUUUGCCGAAUATT dTdT \\
\#3 siRNA & Antisense:dTdTUAUUCGGCAAAUGAGGUGCTT \\
& Sense: GAGGCCUAUAAUGCAAAGATT dTdT \\
& Antisense: dTdTUCUUUGCAUUAUAGGCCUCTT
\end{tabular}

DNMT1, DNA methyltransferases; siRNA, small interfering RNA.

Table II. Primers used for the analysis of mRNA levels by reverse transcription-quantitative PCR.

\begin{tabular}{ll}
\hline Gene & \multicolumn{1}{c}{ Primer sequences (5'-3') } \\
\hline DNMT1 & F: GGCTGAGATGAGGCAAAAAG \\
& R: ACCAACTCGGTACAGGATGC \\
DNMT3 A & F: AGGGAAGACTCGATCCTCGTC \\
& R: GTGTGTAGCTTAGCAGACTGG \\
DNMT3 B & F: GCCTCAATGTTACCCTGGAA \\
& R: CAGCAGATGGTGCAGTAGGA \\
IL-6 & F: TGCAATAACCACCCCTGACC \\
& R: AGCTGCGCAGAATGAGATGA \\
IL-8 & F: GGTGCAGTTTTGCCAAGGAG \\
& R: TTCCTTGGGGTCCAGACAGA \\
GAPDH & F: TCTCCTCTGACTTCAACAGCGACA \\
& R: CCCTGTTGCTGTAGCCAAATCGT
\end{tabular}

IL, interleukin; F, forward; R, reverse.

(Beyotime Institute of Biotechnology). Proteins (30 $\mu \mathrm{g})$ were separated using electrophoresis on $8 \%$ SDS-polyacrylamide gels and transferred to PVDF membranes (EMD Millipore). Next, the membranes were blocked with TBS-Tween 20 (20 mmol Tris-HCl, $150 \mathrm{mmol} \mathrm{NaCl}, 0.05 \%$ Tween-20) containing 5\% BSA (Biofroxx; neoFroxx $\mathrm{GmbH}$ ) for $1 \mathrm{~h}$ at room temperature. Then, the membranes were incubated with primary antibodies against DNMT1 (1:2,000; Abcam), IкB kinase $\alpha \beta$ (IKK $\alpha \beta)$, phosphorylated (p)-IKK $\alpha \beta$, p65, p-p65, $\mathrm{I} \kappa \mathrm{B} \alpha, \mathrm{p}-\mathrm{I} \kappa \mathrm{B} \alpha(1: 1,000 ; \mathrm{NF}-\kappa \mathrm{B}$ Pathway Sampler kit, 9936, Cell Signaling Technology, Inc.), p38, ERK, JNK (1:1,000; MAPK Family Antibody Sampler kit, 9926, Cell Signaling Technology, Inc.), p-p38, p-ERK, p-JNK (1:1,000; phospho-MAPK Family Antibody Sampler kit, 9910, Cell Signaling Technology, Inc.) and GAPDH (1:1,000; Cell Signaling Technology, Inc.) overnight at $4^{\circ} \mathrm{C}$. After rinsing, the membranes were incubated with horseradish peroxidase-conjugated secondary antibodies (1:2,000; AQ160P and AP307P, EMD Millipore) at room temperature for $1 \mathrm{~h}$. An enhanced chemiluminescence system (EMD Millipore) was used to visualize the antibody binding. The relative protein expression levels were normalized to that of the GAPDH gene, and the protein band densities were determined by ImageJ v1.47 software (National Institutes of Health). ReBlot Plus (EMD Millipore) was used to strip and re-probe with the p-antibodies for I $\mathrm{B} \mathrm{B} \alpha$, p38, ERK and JNK to distinguish different target proteins when they share similar molecular weights with the total I $\mathrm{BB} \alpha$, p38, ERK and JNK, respectively, on the same membrane.

ELISA. Human IL-6 ELISA kits (D6050, R\&D Systems, Inc.) and Human IL-8 ELISA kits (D8000C, R\&D Systems, Inc.) were used to analyze the culture supernatant protein concentrations of IL-6 and IL-8 collected after LPS stimulation for $6 \mathrm{~h}$ according to the manufacturer's protocols. A microplate reader (Tecan Safire microplate reader; Tecan Group, Ltd.) was used to evaluate the optical density (OD) values. Based on the standard solution concentration and corresponding OD value, sample concentrations were calculated.

Methylated DNA immunoprecipitation (MeDIP) and $R T-q P C R$. DNA was extracted from hDPCs and fragmented to 200-500-bp fragments with a Bioruptor Waterbath Sonicator ( 8 cycles, $15 \mathrm{sec}$ on/15 sec off, at the highest output level while cooling the tube to $1^{\circ} \mathrm{C}$ in a waterbath). Then, the DNA fragments were diluted to $700 \mu \mathrm{l}$ with TE buffer (Invitrogen; Thermo Fisher Scientific, Inc.) with $60 \mu$ 1 Protein G Magnetic Beads (S1430S; New England BioLabs, Inc.) and denatured for $10 \mathrm{~min}$ at $94^{\circ} \mathrm{C}$. Following denaturation, DNA was immunoprecipitated at $4^{\circ} \mathrm{C}$ overnight with an anti-5mC antibody (1:40, C02010031; Diagenode SA). Then it was incubated for $2 \mathrm{~h}$ with anti-IgG Magnetic Beads (S1430S; New England BioLabs, Inc.) at $4^{\circ} \mathrm{C}$ with agitation. The beads were trapped on a magnetic rack, the supernatant discarded, and washed three times with $1 \mathrm{ml}$ 1XIP buffer [2 mM EDTA, $20 \mathrm{mM}$ Tris ( $\mathrm{pH}=8.0), 1 \%$ Triton $\mathrm{X}-100,0.1 \% \mathrm{SDS}, 150 \mathrm{mM} \mathrm{NaCl}$ ] for $10 \mathrm{~min}$ at $4^{\circ} \mathrm{C}$ with agitation. Beads were then resuspended in $400 \mu \mathrm{l}$ of Elution Buffer $(50 \mathrm{mM}$ Tris- $\mathrm{HCl}, \mathrm{pH}=8.0 ; 10 \mathrm{mM}$ EDTA, $\mathrm{Ph}=8.0 ; 1 \%$ SDS) with $10 \mu \mathrm{l}$ of Proteinase K (Qiagen $\mathrm{GmbH})$. IP with non-specific human IgG was measured as a negative control. After IP, the DNA samples were eluted using phenol-chloroform and precipitated using ethanol. After resuspending the precipitated samples in $10 \mu 1$ Tris buffer, RT-qPCR was performed using $1 \mu \mathrm{l}$ harvested DNA fragments. The primers designed for MeDIP-PCR are shown in Table III.

Statistical analysis. All experiments were carried out at least three times. The data were analyzed by the SPSS 20.0 software 
Table III. Primers used for methylated DNA immunoprecipitation PCR.

\begin{tabular}{ll}
\hline Gene & \multicolumn{1}{c}{ Primer sequences $\left(5^{\prime}-3 '\right)$} \\
\hline IL6 & F: TGGCAGCACAAGGCAAACC \\
& R: GCTTCAGCCCACTTAGAGGAGG \\
IL8 & F: TAGGAAGTGTGATGACTCAGGTT \\
& R: GTCAGAGGAAATTCCACGATT \\
TRAF6 & F: GCTTACTGTAGCCTTGACTGCC \\
& R: GTGGTGCATATCTGTAGTCTCGG \\
MYD88 & F: TTCGCTCACCGACACAGATG \\
& R: GGTCACTGCGGCTGCTCTT \\
\hline
\end{tabular}

IL, interleukin; TRAF6, TNF receptor-associated factor 6; MyD88, myeloid differentiation primary response 88 ; F, forward; R, reverse.

package (IBM Corp.) and are shown as the mean \pm SD. Student's t-test was used to measure the differences between two groups. To evaluate the differences in multiple sets of data, one-way ANOVA or repeated-measures ANOVA with a post hoc Dunnett's test was performed. $\mathrm{P}<0.05$ was considered statistically significant.

\section{Results}

DNMT1 expression in LPS-inflamed hDPCs. To detect the effect of LPS on the inflammatory reaction in hDPCs, hDPCs were stimulated with LPS at a concentration of $1 \mu \mathrm{g} / \mathrm{ml}$ for the indicated times. As illustrated in Fig. 1A and B, compared with the control group, $I L-6$ and $I L-8$ mRNA and protein expression was significantly increased by LPS. IL- 6 and IL- 8 expression was upregulated and peaked after $3 \mathrm{~h}$, which was followed by a gradual decrease. The levels of DNMT1 mRNA were significantly reduced within $24 \mathrm{~h}$ after treatment with LPS. DNMT1 protein expression also decreased, with the most significant change at $3 \mathrm{~h}$ (Fig. 1C and D). Moreover, the mRNA expression of DNMT3a and DNMT3b did not change significantly before or after LPS treatment (Fig. 1).

Effects of DNMT1 on inflammatory cytokine expression in LPS-induced hDPCs. Our preliminary study found that 5-Aza-CdR, a DNMT inhibitor, can increase the secretion of inflammatory cytokines in LPS-stimulated hDPCs, and among the upregulated cytokines, IL- 6 and IL-8 experienced the greatest increase (unpublished data). To investigate the effect of DNMT1-dependent methylation on inflammatory cytokine production in hDPCs stimulated with LPS, the IL-6 and IL-8 expression levels after DNMT1 knockdown in hDPCs transfected with siRNAs were measured. As shown in Fig. 2A and B, after DNMT1 siRNA (\#1, \#2 and \#3) interference, DNMT1 mRNA expression levels were significantly reduced when compared to the negative control group. These data were further confirmed by western blotting, which showed a reduction in the protein expression. Among the DNMTI siRNAs, the siRNA \#1 group showed the best interference effect at $\sim 72 \%$ (Fig. 2B). Therefore, siRNA \#1 was selected as the DNMT1 target sequence for the subsequent experiments.
$I L-6$ and $I L-8$ gene expression levels were then measured in cells stimulated by LPS after DNMT1 depletion (Fig. 2C). The results showed that $I L-6$ and $I L-8$ mRNA expression within $24 \mathrm{~h}$ after LPS stimulation was notably higher in the DNMT1 knockdown group compared with the control group. In LPS-inflamed hDPCs, the protein levels of IL-6 and IL-8 were also significantly increased after DNMT1 knockdown (Fig. 2D).

Effects of DNMT1 on the $N F-\kappa B$ signaling pathway in $L P S$-induced hDPCs. One of the most important signaling pathways that influences inflammatory cytokine production in inflammation induced by LPS is the NF- $\kappa \mathrm{B}$ signaling pathway (25). By means of western blotting, the phosphorylation levels of three crucial proteins of the NF- $\kappa \mathrm{B}$ signaling pathway were examined (IKK $\alpha / \beta$, p65 and I $\kappa \mathrm{B} \alpha$ ) to determine whether DNMT1 is engaged in NF- $\kappa \mathrm{B}$ pathway activation. As illustrated in Fig. 3A and B, DNMT1 knockdown significantly increased the phosphorylation of IKK $\alpha / \beta$ at 15 and $30 \mathrm{~min}$ after LPS treatment in hDPCs. The p 65 and I $\mathrm{B} \alpha$ phosphorylation levels also increased at several time points, but there was no significant difference.

Effects of DNMT1 on the MAPK signaling pathway in $L P S$-induced $h D P C s$. Another vital signaling transduction pathway involved in inflammation in the LPS-related inflammatory response is the MAPK signaling pathway (26). The phosphorylation levels of three key proteins in the MAPK signaling pathway were assessed (p38, ERK1/2 and JNK) to determine whether DNMT1 plays an important role in MAPK signaling pathway activation. As illustrated in Fig. 4A and B, after DNMT1 knockdown in LPS-inflamed hDPCs, the p38 phosphorylation level was increased, while both p-ERK and p-JNK levels were not significantly altered.

Effects of DNMT1 on the dynamic methylation levels of the IL-6, IL-8, TRAF6 and MyD88 gene promoters in $L P S$-induced hDPC inflammation. DNA methylation can regulate the occurrence and progression of inflammatory responses by modulating the methylation levels of inflammation-related cytokines and signaling molecule promoters (27). TRAF6 and MyD88 are key intracellular signal transducers of LPS-induced signaling pathways (28). To identify whether the methylation of $I L-6, I L-8, T R A F 6$ and $M y D 88$ was regulated through DNMT1, the levels of $5 \mathrm{mC}$ present at their gene promoters were examined by means of MeDIP-PCR. The results illustrated that the levels of $5 \mathrm{mC}$ at the $I L-6$ and TRAF 6 promoters decreased notably in LPS-stimulated hDPCs after DNMT1 knockdown. However, no significant change was observed in the $5 \mathrm{mC}$ levels of the $I L-8$ and $M y D 88$ promoters (Fig. 5). These experimental results indicated that DNMT1 can modulate the methylation of IL- 6 and TRAF6 in hDPCs stimulated by LPS.

\section{Discussion}

As a major component of the outer membrane of gram-negative bacteria, LPS serves as the primary pathogenic factor leading to dental pulp inflammation (29). When healthy dental pulp cells are exposed to LPS, pro-inflammatory chemokines and cytokines, including IL-6 and IL-8, are released, 

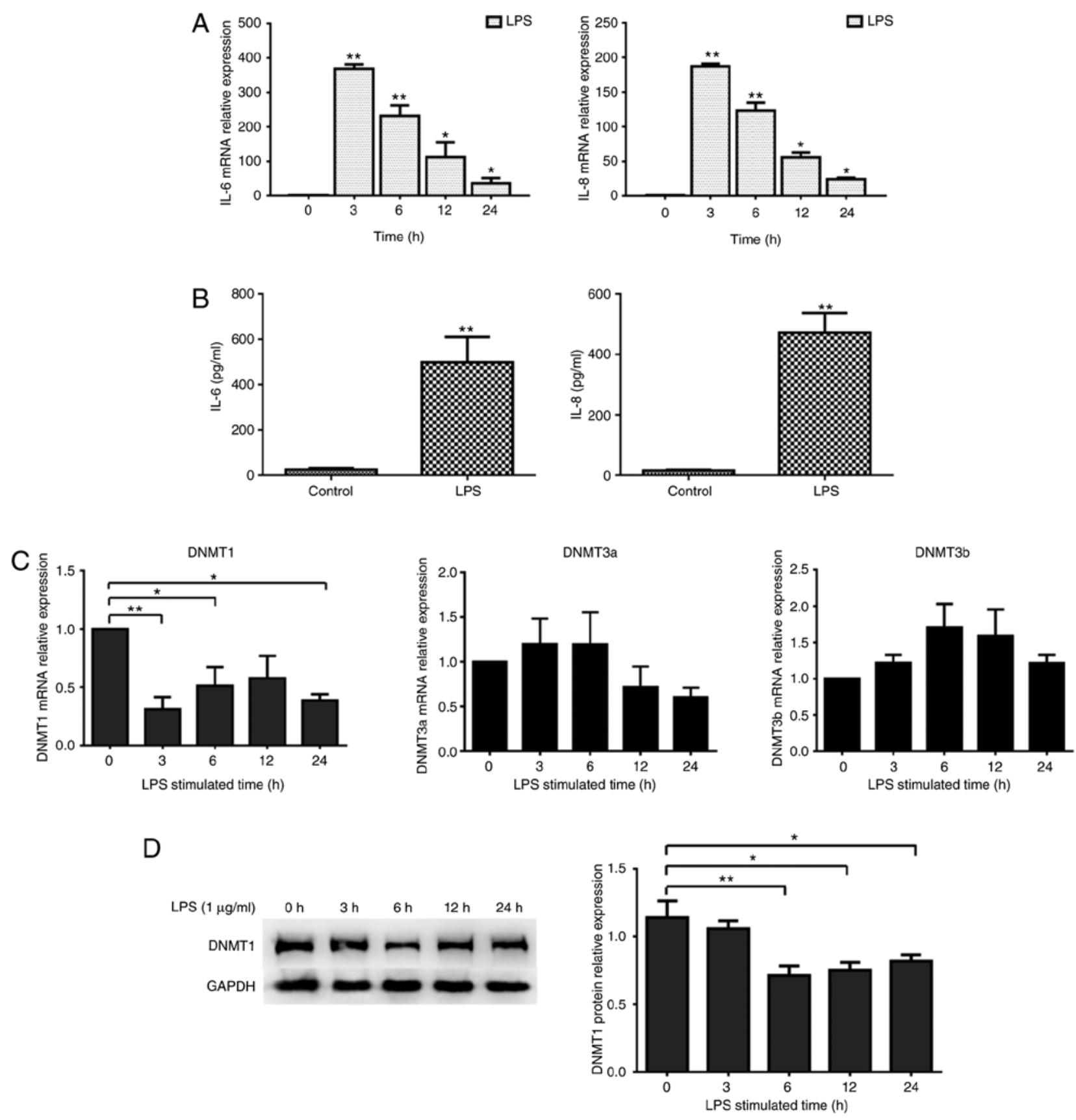

Figure 1. DNMT expression in hDPC inflammatory responses. (A) mRNA expression of $I L-6$ and $I L-8$ in hDPCs was measured by RT-qPCR after stimulation with LPS for 0,3, 6, 12 and $24 \mathrm{~h} ;{ }^{*} \mathrm{P}<0.05 ;{ }^{* *} \mathrm{P}<0.01$. (B) Protein expression levels of IL-6 and IL-8 from the supernatant were detected using ELISA; ${ }^{* *} \mathrm{P}<0.01$. (C) mRNA expression of DNMT1, DNMT3a and DNMT3b was examined using RT-qPCR in hDPCs after the stimulation with LPS for 3, 6, 12 and $24 \mathrm{~h}$. (D) Protein levels of DNMT1 were determined by western blotting after relative quantitative analysis; GAPDH served as the control. Results were presented as the mean $\pm \mathrm{SD}$ of three independent experiments. ${ }^{*} \mathrm{P}<0.05,{ }^{* *} \mathrm{P}<0.01$. DNMT, DNA methyltransferase; hDPCs, human dental pulp cells; RT-qPCR, reverse transcription-quantitative PCR; IL, interleukin; LPS, lipopolysaccharide.

thus triggering subsequent inflammatory events (2). DNA methylation is a major epigenetic regulator that can influence the transcriptional expression of pro-inflammatory cytokines in the initiation and development of the inflammatory response (15-17). However, very little research has sought to define the function of DNA demethylation in the development of the LPS-inflamed dental pulp.

DNA methylation plays a pivotal role in a wide range of inflammatory diseases, and aberrant DNA methylation is often observed in some inflammation-related conditions (30). DNMT1 expression is increased in the rectal epithelium during ulcerative colitis progression in patients and may be a relatively early event in ulcerative colitis-associated tumorigenesis; consequently, this factor may be useful for predicting the risk of colorectal neoplasia in ulcerative colitis (31). In periodontitis, treating human oral keratinocytes with LPS downregulated DNMT1 expression (32). In Sjögren's syndrome, the global DNA methylation level in patient salivary gland epithelial cells was reduced, with a 7-fold decrease in DNMT1 but no significant difference in DNMT3a/b expression (33). To determine the relationship between DNMTs and LPS-inflamed dental pulp, the expression of three DNMTs 

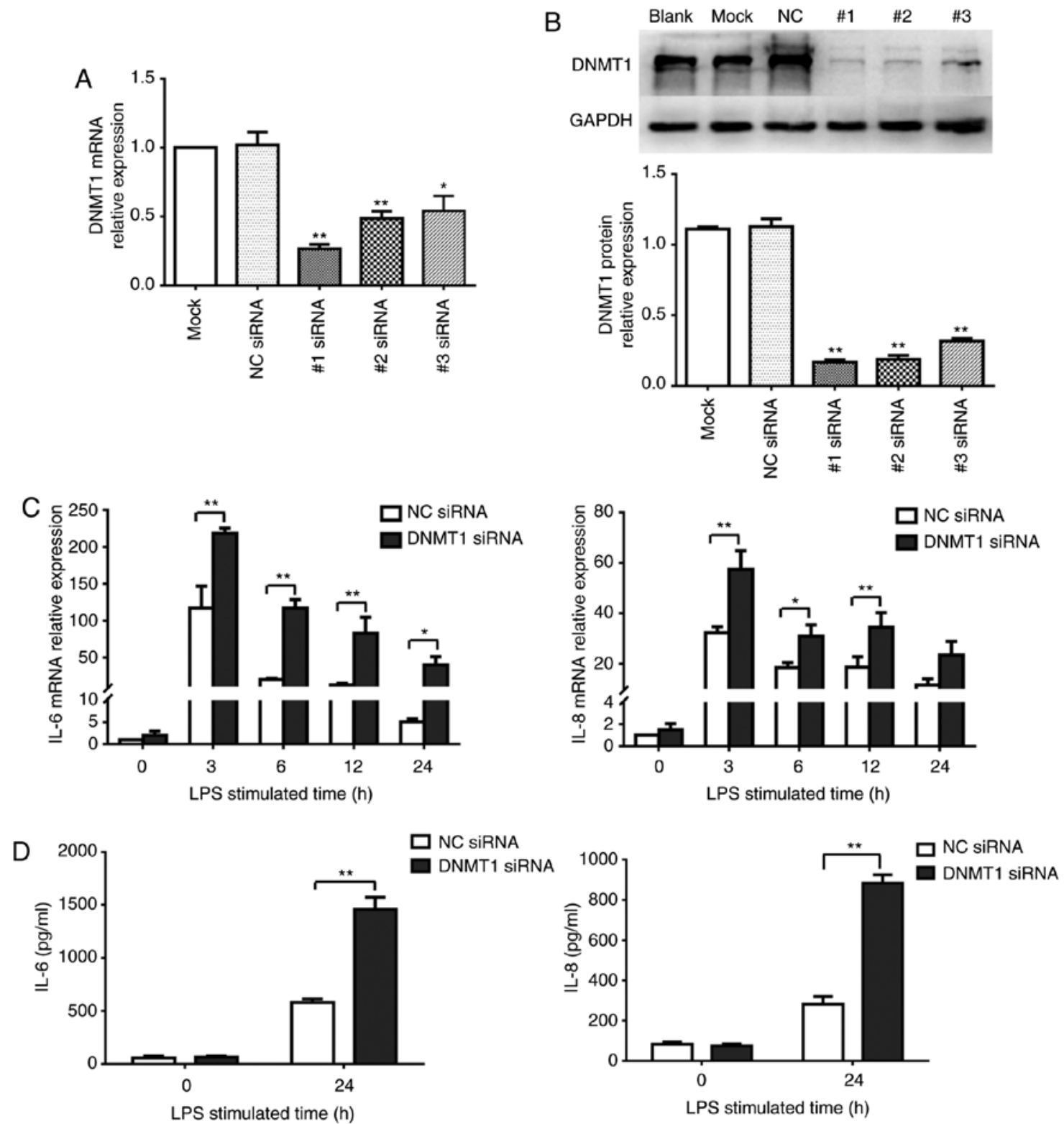

Figure 2. Effects of siRNA-mediated inhibition of DNMT1 expression in hDPCs on LPS-induced inflammatory cytokines. (A) DNMT1 mRNA expression in hDPCs was assessed by RT-qPCR after interference by DNMT1 siRNAs for $48 \mathrm{~h}$ (\#1, \#2 and \#3). (B) Protein expression levels of DNMT1 were examined through western blotting and relative quantitative analysis was performed; GAPDH served as the control. (C) $I L-6$ and $I L-8$ mRNA expression levels were detected through RT-qPCR. (D) IL-6 and IL-8 protein levels were evaluated through ELISA. Results were presented as the mean \pm SD of three independent experiments; ${ }^{*} \mathrm{P}<0.05 ;{ }^{* *} \mathrm{P}<0.01$. siRNA, small interfering RNA; DNMT, DNA methyltransferase; hDPCs, human dental pulp cells; LPS, lipopolysaccharide; RT-qPCR, reverse transcription-quantitative PCR; IL, interleukin; NC, negative control.

in LPS-treated hDPCs were examined. After LPS stimulation, both mRNA and protein expression levels of DNMT1 decreased and reached their lowest level $3 \mathrm{~h}$ after stimulation. In addition, the mRNA expression levels of DNMT3a and $D N M T 3 b$ fluctuated but did not differ significantly. These results suggested that DNMT1-dependent methylation may be involved in the inflammatory progression of dental pulp.

Researchers previously demonstrated that DNA methylation can function as a key epigenetic regulator in the pathogenesis of inflammation-related diseases $(34,35)$. LPS stimulation can induce pro-inflammatory cytokine expression, and the methylation status of their gene promoters is involved in regulating the inflammatory response. In bovine endometrial cells, treatment with LPS can increase $I L-6$ and
IL-8 mRNA expression and decrease the methylation levels of specific CpG sites at the $I L-6$ promoter (at -366 and -660) and the $I L-8$ promoter (at -120 and -48) (35). Treating PBMCs with LPS induces the expression of pro-inflammatory cytokines, including IL-6, TNF- $\alpha$ and IL-1 $\beta$, while also demethylating the IL- 6 gene at the -302 and -264 CpG sites, as well as the TNF- $\alpha$ gene at the $-371 \mathrm{CpG}$ site (36). However, in human intestinal epithelial cells, the $5 \mathrm{CpG}$ sites located near the $I L-8$ transcription start site $(-83,-7,+73,+119$ and +191$)$ were unmethylated on the lower and upper strands in both LPS treated and untreated groups (37). In our previous research, SEQUENOM MassARRAY was used to measure the methylation levels of the $I L-6$ and $I L-8$ promoters in hDPCs after LPS stimulation. The results showed that the methylation level at the $-276 \mathrm{CpG}$ 
A

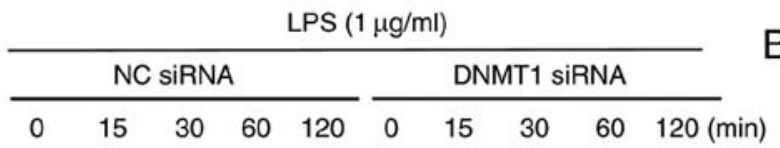

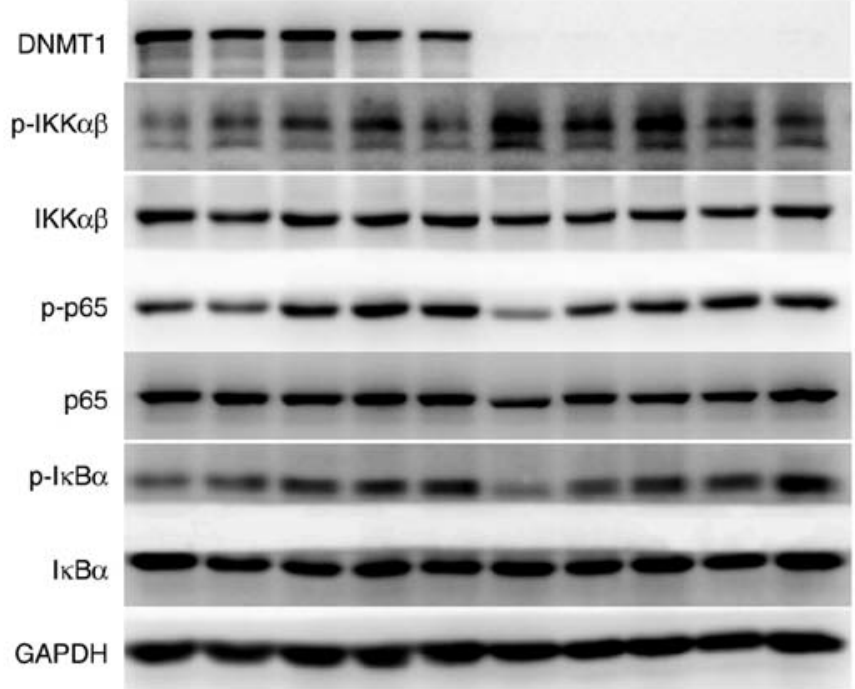

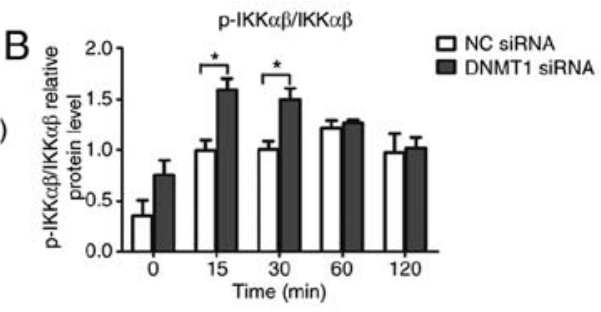
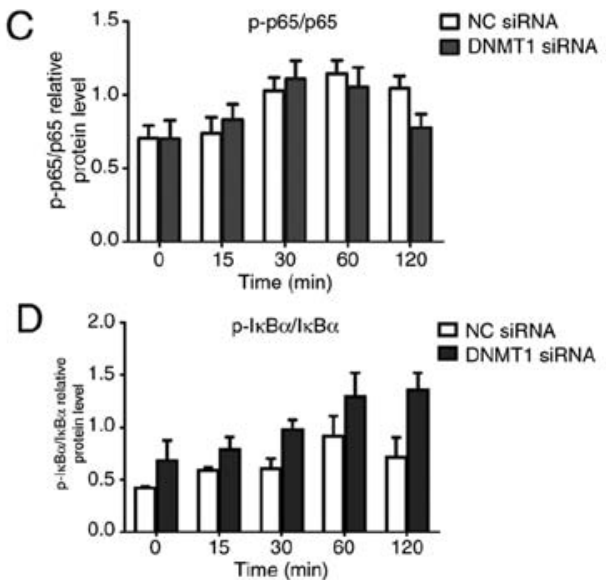

Figure 3. Effects of DNMT1 knockdown on the activation of NF-kB signaling pathways in LPS-induced hDPCs. (A) Expression of key proteins involved in NF- $\mathrm{KB}$ signaling was evaluated using western blotting. The protein samples were collected after a $24 \mathrm{~h}$ siRNA transfection followed by LPS stimulation. GAPDH served as the internal reference. (B-D) Vertical histogram reveals the relative quantitative analysis of the phosphorylation levels of the key proteins in the NF-kB pathway in DNMT1-depleted hDPCs after stimulation with LPS. All results were represented as the mean \pm SD of three independent experiments; ${ }^{*} \mathrm{P}<0.05$. DNMT, DNA methyltransferase; hDPCs, human dental pulp cells; IKK $\alpha \beta$, IкB kinase $\alpha \beta$; LPS, lipopolysaccharide; siRNA, small interfering RNA; NC, negative control; $p$, phosphorylated.

A

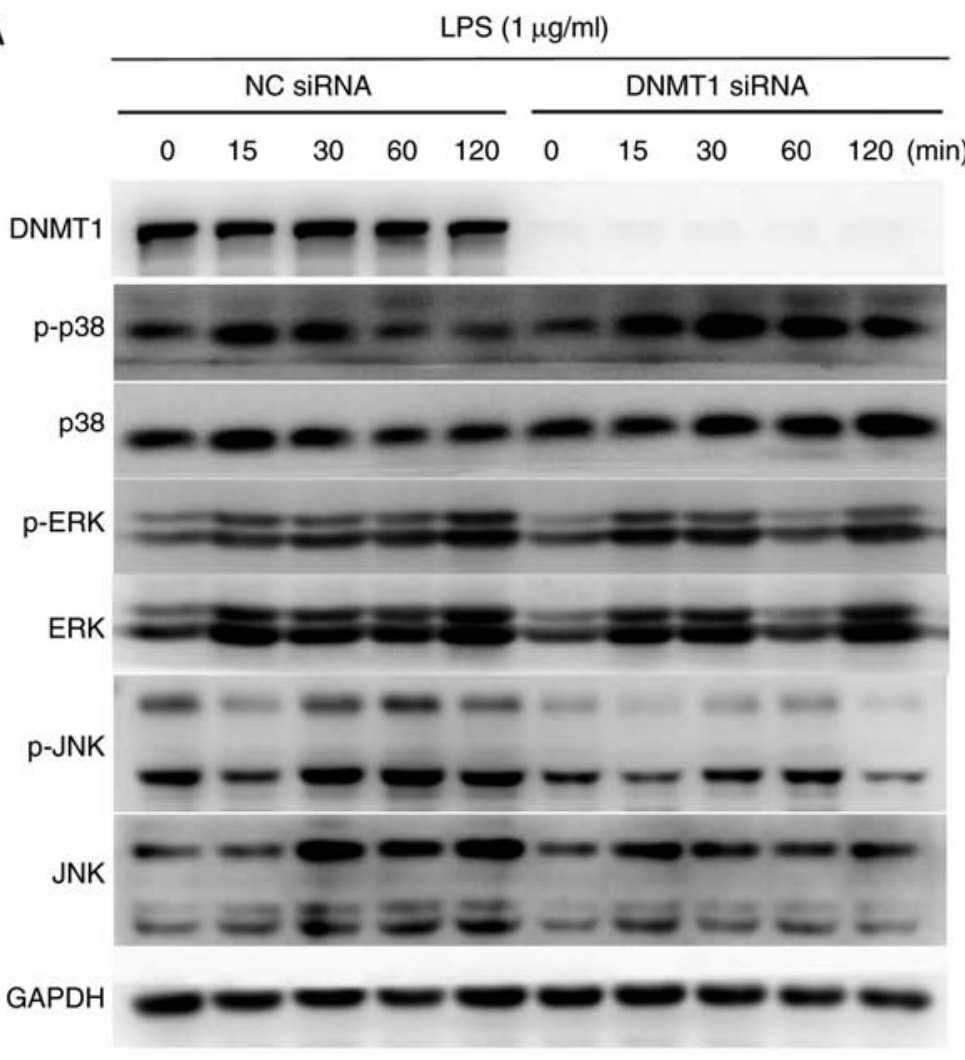

B
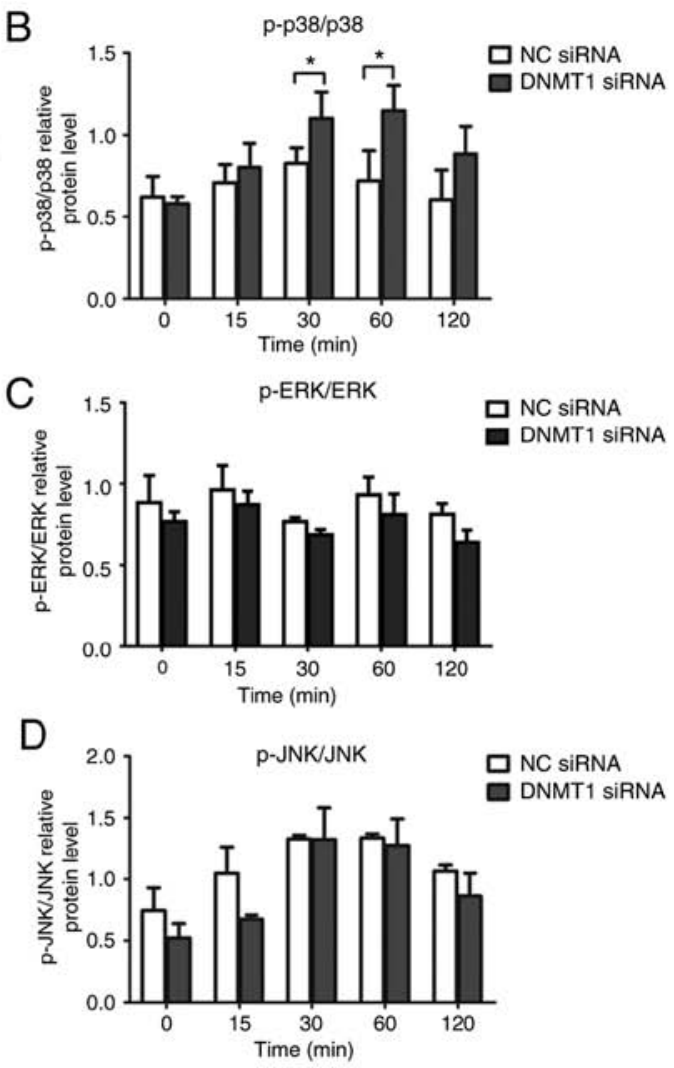

Figure 4. Effects of DNMT1 knockdown on the activation of MAPK signaling pathways in LPS-induced hDPCs. (A) Expression of key proteins in the MAPK signaling pathway was evaluated using western blotting. The protein samples were collected after $24 \mathrm{~h}$ siRNA transfection followed by LPS stimulation. GAPDH served as the internal reference. (B-D) Vertical histogram reveals the relative quantitative analysis of the phosphorylation levels of p38, ERK and JNK in DNMT1-depleted hDPCs after stimulation with LPS. Results were represented as the mean \pm SD of three independent experiments; ${ }^{*} \mathrm{P}<0.05$. DNMT, DNA methyltransferase; hDPCs, human dental pulp cells; LPS, lipopolysaccharide; siRNA, small interfering RNA; NC, negative control; p, phosphorylated. 


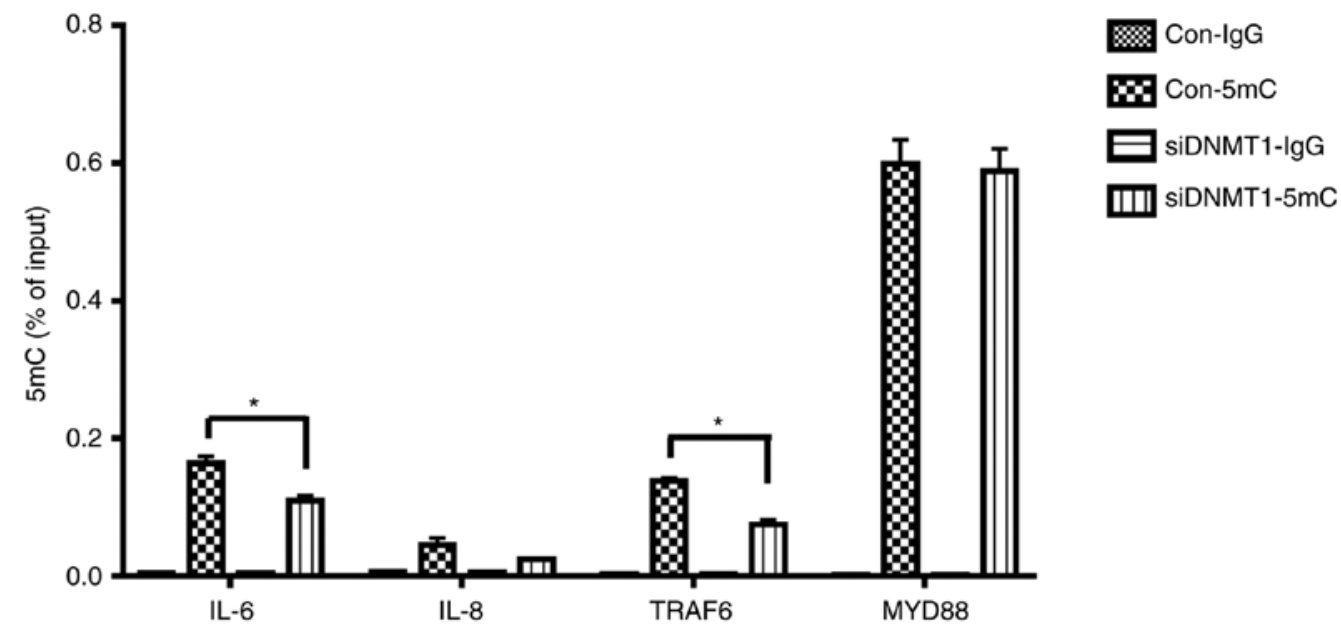

Figure 5. Effects of DNMT1 knockdown on the 5mC levels of the $I L-6, I L-8, M y D 88$ and TRAF6 gene promoters in LPS-induced human dental pulp cells. $I L-6, I L-8, M y D 88$ and TRAF6 gene promoters' dynamic methylation levels were assessed using methylated DNA immunoprecipitation PCR after DNMT1 knockdown, and after $3 \mathrm{~h}$ of stimulation with LPS (control groups). The results were repeated at least three times and presented as the mean \pm SD of three independent experiments; "P<0.05. DNMT, DNA methyltransferase; IL, interleukin; LPS, lipopolysaccharide; 5mC, 5-methylcytosine; TRAF6, TNF receptor-associated factor 6; MyD88, myeloid differentiation primary response 88; si, small interfering (RNA).

site in the $I L-6$ promoter decreased after LPS stimulation. However, there was no difference in the methylation level of the $I L-8$ promoter (unpublished data). In the present study, to investigate the function of DNMT1 in inflammatory cytokine production by hDPCs after LPS stimulation, DNMT1 knockdown in hDPCs was established through siRNA transfection. The expression of DNMT1 was significantly decreased following depletion of DNMT1, which is consistent with our previous research $(20,38)$. Next, the LPS-stimulated cytokine expression after knocking down DNMT1 was examined. $D N M T 1$ silencing prominently enhanced the production of the cytokines IL-6 and IL-8, thereby indicating that DNMT1 may be a regulator that negatively targets cytokine accumulation in hDPCs inflamed by LPS.

It is commonly known that the MAPK and NF- $\mathrm{KB}$ signaling pathways play critical roles in mediating inflammatory reactions and are likely regulated by DNA methylation $(19,39)$. In aged mouse macrophages, phosphorylation of I $\kappa \mathrm{B} \alpha$ in the NF- $\kappa \mathrm{B}$ signaling pathway was increased after treatment with the demethylation agent 5-Aza-CdR (40). 5-Aza-CdR also increased I $\mathrm{I} B \alpha$ and IKK $\alpha / \beta$ phosphorylation levels to promote the activation of NF- $\kappa \mathrm{B}$ signaling in gastric cancer cells (41). A study on lung tissue inflammation revealed that 5-Aza-CdR can markedly decrease p38, JNK and ERK phosphorylation levels, thereby inhibiting MAPK signaling pathway activation under LPS stimulation (19). The levels of DNA methylation were affected in 27 gene promoters of the MAPK pathway in PBMCs and plasma samples from children who were constantly exposed to air pollutants (42). To explore whether DNA methylation influences the signaling pathways in LPS-treated hDPCs, the phosphorylation levels of several important signaling molecules in the MAPK and NF- $\mathrm{KB}$ signaling pathways were examined. The data from the present study showed that compared to LPS exposure alone, DNMTI depletion upregulated the phosphorylation levels of IKK $\alpha / \beta$ in the NF- $\kappa B$ signaling pathway and the phosphorylation level of $\mathrm{p} 38$ in the MAPK signaling pathway. Therefore, DNMT1 suppressed both the MAPK and NF- $\kappa \mathrm{B}$ signaling pathways in
LPS-stimulated hDPCs, further confirming that DNMT1 acts as a negative regulator in inflamed hDPCs.

Previous studies have proposed that DNA methylation not only affects the methylation level of inflammatory cytokine promoters, but also changes the methylation status of intracellular signal transducers of signaling pathways (43). TRAF6 and MyD88, key intracellular signal transducers of the MAPK and NF- $\mathrm{\kappa B}$ signaling pathways, can be regulated by DNA methylation $(20,21)$. TRAF6 hypermethylation has been linked to low TRAF6 gene expression levels in PBMCs during inflammatory bowel diseases (21). In addition, MyD88 was shown to have consistently higher methylation levels in its promoter region in moderate localized aggressive periodontitis (LAP) than in severe LAP (44). In patients with LAP, the methylation level of the MyD88 promoter is negatively associated with several cyto/chemokines, such as IL-8 and IL-6 (44). In the present study, to explore whether these signal transduction factors are regulated by DNA methylation in LPS-treated hDPCs, MeDIP and RT-qPCR were used to analyze the dynamic $5 \mathrm{mC}$ levels of the $I L-6, I L-8, T R A F 6$ and $M y D 88$ gene promoters in DNMT1-deficient cells. Notably, the $5 \mathrm{mC}$ levels of the $I L-6$ and TRAF6 gene promoters decreased, suggesting that $D N M T 1$ knockdown downregulated $5 \mathrm{mC}$ at the $I L-6$ and TRAF6 gene promoters. Although a modest decrease in the $I L-8$ and $M y D 88$ gene promoter $5 \mathrm{mC}$ levels was observed, there were no significant differences. These observations indicated that DMNT1 can mediate the $5 \mathrm{mC}$ level of $I L-6$ and TRAF6 in LPS-inflamed hDPCs.

In summary, the present study showed that stimulating hDPCs with LPS decreased the expression of the DNA methyltransferase DNMT1.DNMT1 depletion increased LPS-induced cytokine secretion in hDPCs, and activated NF- $\mathrm{KB}$ and MAPK signaling. Furthermore, silencing DNMT1 was involved in downregulating methylation levels at the promoters of $I L-6$ and TRAF6. This study indicated that DNMT1-dependent DNA methylation plays a role in the inflammatory response of hDPCs stimulated by LPS, and provides a novel rationale for researchers to further reveal the molecular mechanisms of inflamed dental pulp. 


\section{Acknowledgements}

Not applicable.

\section{Funding}

The present study was financially supported by the National Natural Science Foundation of China (grant no. 81771058).

\section{Availability of data and materials}

The datasets used and/or analyzed during the current study are available from the corresponding author on reasonable request.

\section{Authors' contributions}

QX designed the study and provided scientific leadership to junior colleagues. LC and MZ performed the experiments and statistically analyzed the results. LC wrote the manuscript. QL and DL analyzed data, providing constructive comments. All authors read and approved the final manuscript.

\section{Ethics approval and consent to participate}

The present study was authorized by the institutional Ethical Review Boards of the Guanghua School of Stomatology of Sun Yat-sen University, and written informed consent for this investigation was provided from all patients who participated in the experiment in the study.

\section{Patient consent for publication}

Not applicable.

\section{Competing interests}

The authors declare that they have no competing interests.

\section{References}

1. Bindal P, Ramasamy TS, Kasim NHA, Gnanasegaran N and Chai WL: Immune responses of human dental pulp stem cells in lipopolysaccharide-induced microenvironment. Cell Biol Int 42: 832-840, 2018

2. Renard E, Gaudin A, Bienvenu G, Amiaud J, Farges JC Cuturi MC, Moreau A and Alliot-Licht B: Immune cells and molecular networks in experimentally induced pulpitis. J Dent Res 95: 196-205, 2016.

3. Li JG, Lin JJ, Wang ZL, Cai WK, Wang PN, Jia Q, Zhang AS, Wu GY, Zhu GX and Ni LX: Melatonin attenuates inflammation of acute pulpitis subjected to dental pulp injury. Am J Transl Res 7: 66-78, 2015.

4. Feng Z, Li Q, Meng R, Yi B and Xu Q: METTL3 regulates alternative splicing of MyD88 upon the lipopolysaccharide-induced inflammatory response in human dental pulp cells. J Cell Mol Med 22: 2558-2568, 2018.

5. Song F, Sun H, Wang Y, Yang H, Huang L, Fu D, Gan J and Huang C: Pannexin3 inhibits TNF- $\alpha$-induced inflammatory response by suppressing NF- $\kappa B$ signaling pathway in human dental pulp cells. J Cell Mol Med 21: 444-455, 2017.

6. Hui T, A P, Zhao Y, Yang J, Ye L and Wang C: EZH2 regulates dental pulp inflammation by direct effect on inflammatory factors. Arch Oral Biol 85: 16-22, 2018.

7. Hui T, Wang C, Chen D, Zheng L, Huang D and Ye L: Epigenetic regulation in dental pulp inflammation. Oral Dis 23: 22-28, 2017.
8. Bei Y, Tianqian H, Fanyuan Y, Haiyun L, Xueyang L, Jing Y, Chenglin W and Ling Y: ASH1L suppresses matrix metalloproteinase through mitogen-activated protein kinase signaling pathway in pulpitis. J Endod 43: 306.e2-314.e2, 2017.

9. Cardoso FP, Viana MB, Sobrinho AP, Diniz MG, Brito JA, Gomes CC, Moreira PR and Gomez RS: Methylation pattern of the IFN-gamma gene in human dental pulp. J Endod 36: 642-646, 2010

10. Smith ZD and Meissner A: DNA methylation: Roles in mammalian development. Nat Rev Genet 14: 204-220, 2013.

11. Moore LD, Le T and Fan G: DNA methylation and its basic function. Neuropsychopharmacology 38: 23-38, 2013.

12. Loo SK, Ab Hamid SS, Musa M and Wong KK: DNMT1 is associated with cell cycle and DNA replication gene sets in diffuse large B-cell lymphoma. Pathol Res Pract 214: 134-143, 2018.

13. Auclair G, Guibert S, Bender A and Weber M: Ontogeny of CpG island methylation and specificity of DNMT3 methyltransferases during embryonic development in the mouse. Genome Biol 15: $545,2014$.

14. Kettunen E, Hernandez-Vargas H, Cros MP, Durand G, Le Calvez-Kelm F, Stuopelyte K, Jarmalaite S, Salmenkivi K, Anttila S, Wolff H, et al: Asbestos-associated genome-wide DNA methylation changes in lung cancer. Int J Cancer 141: 2014-2029, 2017.

15. Qiu J, Zhang YN, Zheng X, Zhang P, Ma G and Tan H: Notch promotes DNMT-mediated hypermethylation of Klotho leads to COPD-related inflammation. Exp Lung Res 44: 368-377, 2018.

16. Shen J, Wu S, Guo W, Liang S, Li X and Yang X: Epigenetic regulation of pro-inflammatory cytokine genes in lipopolysaccharide-stimulated peripheral blood mononuclear cells from broilers. Immunobiology 222: 308-315, 2017.

17. Cheng C, Huang C, Ma TT, Bian EB, He Y, Zhang L and Li J: SOCS1 hypermethylation mediated by DNMT1 is associated with lipopolysaccharide-induced inflammatory cytokines in macrophages. Toxicol Lett 225: 488-497, 2014.

18. Ma SC, Hao YJ, Jiao Y, Wang YH, Xu LB, Mao CY, Yang XL, Yang AN, Tian J, Zhang MH, et al: Homocysteine-induced oxidative stress through TLR4/NF- $\kappa \mathrm{B} / \mathrm{DNMT1}$-mediated LOX-1 DNA methylation in endothelial cells. Mol Med Rep 16: 9181-9188, 2017.

19. Huang X, Kong G, Li Y, Zhu W, Xu H, Zhang X, Li J, Wang L, Zhang Z, Wu Y, et al: Decitabine and 5-azacitidine both alleviate LPS induced ARDS through anti-inflammatory/antioxidant activity and protection of glycocalyx and inhibition of MAPK pathways in mice. Biomed Pharmacother 84: 447-453, 2016.

20. Meng R, Li D, Feng Z and Xu Q: MyD88 hypermethylation mediated by DNMT1 is associated with LTA-induced inflammatory response in human odontoblast-like cells. Cell Tissue Res 376: 413-423, 2019.

21. McDermott E, Ryan EJ, Tosetto M, Gibson D, Burrage J, Keegan D, Byrne K, Crowe E, Sexton G, Malone K, et al: DNA methylation profiling in inflammatory bowel disease provides new insights into disease pathogenesis. J Crohns Colitis 10: 77-86, 2016.

22. Gronthos S, Mankani M, Brahim J, Robey PG and Shi S: Postnatal human dental pulp stem cells (DPSCs) in vitro and in vivo. Proc Natl Acad Sci USA 97: 13625-13630, 2000.

23. Jung JY, Woo SM, Kim WJ, Lee BN, Nör JE, Min KS, Choi CH, Koh JT, Lee KJ and Hwang YC: Simvastatin inhibits the expression of inflammatory cytokines and cell adhesion molecules induced by LPS in human dental pulp cells. Int Endod J 50: 377-386, 2017.

24. Livak KJ and Schmittgen TD: Analysis of relative gene expression data using real-time quantitative PCR and the 2(-Delta Delta $\mathrm{C}(\mathrm{T})$ ) method. Methods 25: 402-408, 2001.

25. Liu J, Guo S, Jiang K, Zhang T, Zhiming W, Yaping Y, Jing Y, Shaukat A and Deng G: miR-488 mediates negative regulation of the AKT/NF- $\kappa \mathrm{B}$ pathway by targeting Racl in LPS-induced inflammation. J Cell Physiol, 2019.

26. Shang L, Wang T, Tong D, Kang W, Liang Q and Ge S: Prolyl hydroxylases positively regulated LPS-induced inflammation in human gingival fibroblasts via TLR4/MyD88-mediated AKT/NF- $\kappa$ B and MAPK pathways. Cell Prolif 51: e12516, 2018.

27. Matt SM, Lawson MA and Johnson RW: Aging and peripheral lipopolysaccharide can modulate epigenetic regulators and decrease IL-1 $\beta$ promoter DNA methylation in microglia. Neurobiol Aging 47: 1-9, 2016.

28. Wang Q, Zhou X, Zhao Y, Xiao J, Lu Y, Shi Q, Wang Y, Wang H and Liang Q: Polyphyllin I ameliorates collagen-induced arthritis by suppressing the inflammation response in macrophages through the NF- $\kappa$ B pathway. Front Immunol 9: 2091, 2018. 
29. Love RM and Jenkinson HF: Invasion of dentinal tubules by oral bacteria. Crit Rev Oral Biol Med 13: 171-183, 2002.

30. Barnicle A, Seoighe C, Greally JM, Golden A and Egan LJ: Inflammation-associated DNA methylation patterns in epithelium of ulcerative colitis. Epigenetics 12: 591-606, 2017.

31. Fujii S, Katake Y and Tanaka H: Increased expression of DNA methyltransferase-1 in non-neoplastic epithelium helps predict colorectal neoplasia risk in ulcerative colitis. Digestion 82: 179-186, 2010.

32. de Camargo Pereira G, Guimarães GN, Planello AC, Santamaria MP, de Souza AP, Line SR and Marques MR: Porphyromonas gingivalis LPS stimulation downregulates DNMT1, DNMT3a, and JMJD3 gene expression levels in human HaCaT keratinocytes. Clin Oral Investig 17: 1279-1285, 2013.

33. Thabet Y, Le Dantec C, Ghedira I, Devauchelle V, Cornec D, Pers JO and Renaudineau Y: Epigenetic dysregulation in salivary glands from patients with primary Sjögren's syndrome may be ascribed to infiltrating B cells. J Autoimmun 41: 175-181, 2013

34. Hedrich CM and Tsokos GC: Epigenetic mechanisms in systemic lupus erythematosus and other autoimmune diseases. Trends Mol Med 17: 714-724, 2011.

35. Wang J, Yan X, Nesengani LT, Ding H, Yang L and Lu W: LPS-induces IL-6 and IL-8 gene expression in bovine endometrial cells 'through DNA methylation'. Gene 677: 266-272, 2018.

36. Angrisano T, Pero R, Peluso S, Keller S, Sacchetti S, Bruni CB, Chiariotti L and Lembo F: LPS-induced IL-8 activation in human intestinal epithelial cells is accompanied by specific histone $\mathrm{H} 3$ acetylation and methylation changes. BMC Microbiol 10: 172, 2010

37. Shen J, Liu Y, Ren X, Gao K, Li Y, Li S, Yao J and Yang X: Changes in DNA methylation and chromatin structure of pro-inflammatory cytokines stimulated by LPS in broiler peripheral blood mononuclear cells. Poult Sci 95: 1636-1645, 2016.
38. Mo Z, Li Q, Cai L, Zhan M and Xu Q: The effect of DNA methylation on the miRNA expression pattern in lipopolysaccharide-induced inflammatory responses in human dental pulp cells. Mol Immunol 111: 11-18, 2019

39. Jangiam $\mathrm{W}$, Tungjai $\mathrm{M}$ and Rithidech $\mathrm{KN}$ : Induction of chronic oxidative stress, chronic inflammation and aberrant patterns of DNA methylation in the liver of titanium-exposed CBA/CaJ mice. Int J Radiat Biol 91: 389-398, 2015.

40. Jiang M, Xiang Y, Wang D, Gao J, Liu D, Liu Y, Liu S and Zheng D: Dysregulated expression of miR-146a contributes to age-related dysfunction of macrophages. Aging Cell 11: 29-40, 2012.

41. Kim TW, Lee SJ, Oh BM, Lee H, Uhm TG, Min JK, Park YJ, Yoon SR, Kim BY, Kim JW, et al: Epigenetic modification of TLR4 promotes activation of $N F-\kappa B$ by regulating methyl-CpG-binding domain protein 2 and $\mathrm{Sp} 1$ in gastric cancer. Oncotarget 7: 4195-4209, 2016.

42. Carmona JJ, Sofer T, Hutchinson J, Cantone L, Coull B, Maity A, Vokonas P, Lin X, Schwartz J and Baccarelli AA: Short-term airborne particulate matter exposure alters the epigenetic landscape of human genes associated with the mitogen-activated protein kinase network: A cross-sectional study. Environ Health 13: 94, 2014.

43. Wang X, Feng Z, Li Q, Yi B and Xu Q: DNA methylcytosine dioxygenase ten-eleven translocation 2 enhances lipopolysaccharide-induced cytokine expression in human dental pulp cells by regulating MyD88 hydroxymethylation. Cell Tissue Res 373: 477-485, 2018.

44. Shaddox LM, Mullersman AF, Huang H, Wallet SM, Langaee T and Aukhil I: Epigenetic regulation of inflammation in localized aggressive periodontitis. Clin Epigenetics 9: 94, 2017. 